\title{
Aligning Web content and organisational strategy: Towards increasing funding
}

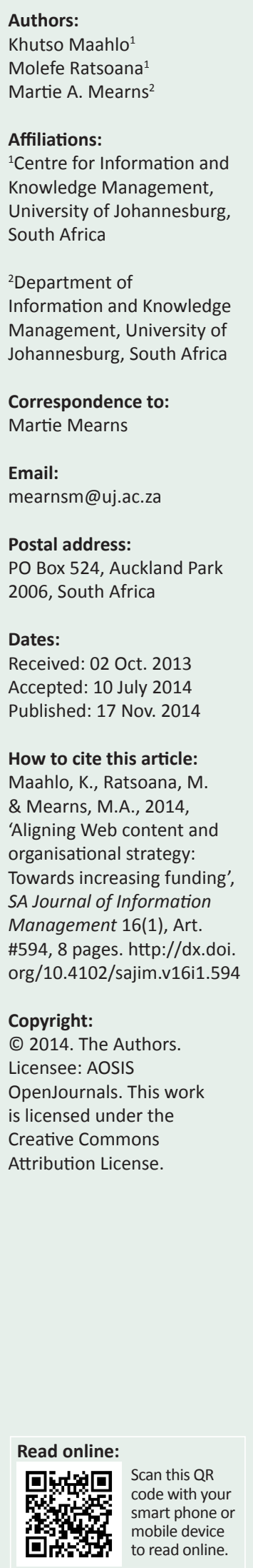

Background: The effectiveness with which the Aurum Institute manages to communicate its organisational strategic objectives through its website was investigated. Despite its ground breaking research and programs which have made a positive impact on the community, the major problem that it faces is funding its core.

Objectives: An investigation of the website's content was carried out to determine the extent to which the organisational strategy is reflected to potential funders visiting the site. Requirements for aligning the content with the organisational strategy were identified.

Method: Content analysis was used where secondary data, such as website content and organisational strategic objectives, was analysed. An interview was also conducted with Aurum's knowledge manager to gain an in-depth understanding of the organisation's strategic objectives and to validate the initial findings.

Results: The results show that the website content was not effectively representative of Aurum's strategic objectives, and that the website structure did not effectively share the information that supports the decision-making process of potential investors. Recommendations were presented to the organisation in the form of a prototype website which reflects the desired website content that is representative of Aurum's strategic objectives.

Conclusion: Further research is required in determining the effect that a strategically driven website could possibly exert on the funding potential of the organisation. The theoretical approach used in this study can be used as a scoping exercise in organisations of a similar business nature.

\section{Introduction}

Website content that is effectively integrated with the strategic objectives of an organisation uncovers opportunities of increasing its audience base, specifically in the case where the website is geared towards drawing the attention of potential investors or funding opportunities. According to Akakandelwa (2011:421), websites act as connection and communication points for information users with electronic information. As a result, most corporations, organisations or institutions are making efforts to launch themselves into the virtual world of the World Wide Web. Rocha (2012:374) explains online perspective of organisations today, that they: '(I)nvest time and money to develop and maintain their website's quality. These websites should establish an effective information and communication channel between organisations and their clients'.

\section{Panopoulou, Tambouris and Tarabanis (2008) note the following:}

Literature suggests that the sophistication of websites is related to a number of characteristics and features regarding interface and aesthetics, navigation, consistency and patterns, content relevancy and accuracy, accessibility, security and currency of information. (p. 518)

However, these characteristics and features need to 'be reviewed regularly to determine whether they are fulfilling the reasons for which they were developed' (Chiou, Lin \& Perng 2011:1463).

It has been identified that, despite the recognised importance of having a website, most organisations do not plan properly for it. Nacar and Burnaz (2011:276) and Liao, Proctor and Salvendy (2008:44) highlight that 'content preparation could be handled from two main perspectives: the information content to be provided and the structure of how this information is to be presented'. The information content and structure need to be addressed in the website strategy of the organisation. Organisations also make use of website strategies to communicate and collaborate with stakeholders and inform them of the products and services that the organisation must provide (Chiou, Lin \& Perng 2010:286). The website strategy development process ensures that an organisation addresses the strategic objectives that the website should achieve, one of which should be to effectively communicate its vision, mission and objectives, together with evidence that supports these aims, to its users. 
It has become increasingly important that an organisation operates from a business strategy that ensures the efficient allocation of resources to relevant tasks. This is even more important for non-profit organisations (NPOs) because their funding comes from external sources. The same principle applies to their website strategies. NPOs are required to effectively communicate their mission and vision to the users of their website by certifying that their strategic objectives are well communicated. It is the purpose of this article to report on research conducted at the Aurum Institute. The Aurum Institute is an NPO based in South Africa whose aim is to improve on the health of the community through innovation in Tuberculosis and HIV and AIDS research (Aurum Institute 2012:3). Prof Gavin Churchyard, CEO of the Aurum Institute stated in the annual report of 2010 that 'one of the major problems that face us is funding our core' (Aurum Institute 2010:45). Aurum owns a website on which interested parties and potential funders can access information about the institute and its research initiatives. In addressing the above-mentioned funding issue, Aurum's website content was assessed to establish if it is effectively aligned to the strategic objectives of the organisation.

An organisation can make use of a website as a medium which will be accessed to transfer information and knowledge, and facilitate positive outcomes of the decision-making process made by the investors (BouLlusar \& Segarra-Cipres 2006:106). The website designers need to analyse the content using relevant analysis tools to ensure that it is of sound quality, relevant, timely and that it facilitates decision-making. The organisation needs to identify the content it wishes to present on the website which will appeal to the targeted audience. Bill Gates (1996) also emphasised the power of persuasion that content possesses. He states that the quality and presentation of the content has the power to lure or push the audience away. In this context it is argued that a conscious inclusion of organisational strategy into website content is required to enhance Aurum Institute's credibility, strengthen its organisational relations and increase its competitive advantage for better funding opportunities.

This argument is based on the findings generated through the investigation that addressed the following main research question:

How effective is the Aurum Institute's website content in portraying their strategic objectives towards potentially attracting external funding or sponsorships?

In order to have addressed this research question the study was guided by the following sub-questions:

- What is the content quality and condition of the current website of the Aurum Institution?

- What are the organisational goals, objectives, values and strategy of the Aurum Institution and how are they currently represented by the website?
- What needs to be carried out to ensure that the Aurum Institute's content is aligned with its objectives?

- Using the findings from the above sub-questions, what website content will be suitable for the implementation of a website that will represent organisational goals and attract investments?

A qualitative approach was followed to collecting and analysing data, collecting and analysing secondary data as well as to conducting an in-depth interview. The secondary data comprised of Aurum's knowledge audit that was completed a year prior to the commencement of this study, and the inclusion of the current website content and the organisation's strategic objectives. A structured interview took place with the knowledge manager of Aurum Institute to fill the data gaps identified. All results were analysed and recommendations derived and integrated in the new prototype website.

\section{Literature review}

Dias and Reinhard (2004:5) maintain that organisations can add value to the business processes and activities by understanding the importance of Internet technologies and their platforms. Bailey (2010) mentions Gates (1996) who claimed that organisations could facilitate the management of information and the creation of quality website content, which will result in added value to the processes, procedures, communication and collaboration channels of those organisations. Chiou et al. (2010:286) stated that implementing a website strategy within the organisation can increase communication and collaboration with its stakeholders and customers. Moustakis, Tsironis and Litos (2006:60) identified the important factors that characterise websites of good quality; these include the following:

- content

- navigation

- structure and design

- look and feel

- multimedia used.

Huizingh (2000:124) emphasised the importance of the structure and quality of content as a result of the vital role they play in persuading stakeholders and customers.

The nature of Aurum's business necessitates that the organisation attracts potential investors to ensure that projects are well resourced and are effectively executed. De Troyer (1998:2) identified that users experience problems with organisations' websites; these include, amongst others, unclear mission statements of the organisation, no clearly defined target market and poor management of content. An organisation can increase the satisfaction levels of its stakeholders and increase revenue through effective information and knowledge sharing with them (Mpindiwa 2010:4). According to Hernandez, Jimenez and Martin (2009:362) a large number of studies in the 1990s placed much emphasis on website design (the content, use and objective of 
the website) but failed to identify the factors that improve the organisation's website strategy. It is imperative that the organisation understands the website strategy as well as its purpose in the organisation's communication plan before it is integrated.

\section{Website strategy}

According to Hernandez et al. (2009:364) organisations engaged in electronic commerce aided by an effective website strategy have the potential of being exposed to a larger market than organisations that do not effectively incorporate website strategy. Schmidt, Cantallops and Dos Santos (2008:504) stated that the implementation of the website strategy in the organisation enhances communication with stakeholders, and improves marketing mix and business practices. The website strategy is utilised by individuals or organisations to inform their audiences about the services and products offered by the organisation (Chiou et al. 2010:282). As part of their website strategy it is important that organisations identify the characteristics that enhance the interaction and relationship of the organisation with its stakeholders and customers (Baloglu \& Pekcan 2006:174). Hernandez et al. (2009:364) identified these characteristics as the factors that are inherent in the Web Index Assessment (WIA) which facilitate effective and efficient access to the organisation's information through the website. Below is a brief summary of the factors that improve the quality of a website:

- Accessibility: this term refers to the ability of potential and current users to identify and access the website and its content. The volume of traffic that reaches the website influences the accessibility of the content on the website. There are two indicators that measure accessibility to the website; these are the position of the website on the search engine results and the popularity of the website. Search Engine Optimisation (SEO) and Search Engine Marketing (SEM) are tools that are used to measure the accessibility of the website. The SEO tool works to improve the traffic of the website using the search engines by submitting targeted keywords. The SEM tool works to obtain free search listings from the search engines by purchasing the search listings. It aims at increasing the traffic by improving the website's visibility on the search engines.

- Speed: this factor plays a vital role in the satisfaction levels of users and facilitates effective transactional processes on the virtual space. Website designers need to ensure that the loading time of the website is fast, and failure to do so will lead to a potential loss of users searching for information to other websites. Websites with a slow loading time increases the possibility of pushing potential customers away. Hernandez et al. (2009:364) stated that if a website takes more than 10 seconds to load, the website has a high chance of losing the user.
- Navigability: website designers need to put in place applications that will ensure ease of use and ability to find the content on the website. Poorly designed websites may result in inaccessibility to valuable information which could have the potential of dissuading stakeholders or customers to invest in or purchase into the organisation. Hernandez et al. (2009:364) further identifies the factors that website designers should consider in the design of the website which will enable users to successfully navigate through the website. Firstly, the content should be classified and organised according to a relevant topic and headings; secondly, the information should be labeled; thirdly, the navigation system should be designed in such a way that it will guide the user through the content and fourthly, the users must be guided to the information they need in order to make decisions.

- Content quality: the basic goal of implementing a website strategy is to provide information that is relevant to the organisation. There are two functions, or roles, that content plays on the website. (1) It informs the audience about the organisation's background, the products and services it offers, and the vision of the organisation. The information should be regularly updated and relevant. It should also be precise to ensure that a user has a full understanding of the organisation without being subjected to information overload. (2) It facilitates communication and interaction between the organisation and the customers. Website designers need to create a communication medium that will be used to share content with the customers and also interact with them (Hernandez et al. 2009:364).

By identifying the above characteristics an organisation can determine the website features that are important to incorporate into its website strategy.

Grigoroudis et al. (2008:1346) explain the following about quality and communication: Organisations invest time and money to develop and maintain their website's quality. These websites should establish an effective information and communication channel between organisations and their clients.

Hussainey and Mouselli (2010:6) maintain that stakeholders need to be well informed in order to make good decisions regarding purchases or investments in the organisation. Stakeholders are persuaded by the aesthetics and the manner in which the content is displayed on the website when they decide about investing in the organisation (Hussaney \& Mouseli 2010:6). Huizingh (2000:124) claims that the engagement of stakeholders with the organisation is dependent on the information shared by the organisation, together with its ability to communicate the potential growth and return on investment of the organisation. Organisations that are non-profit in nature depend on financial assistance to successfully implement their projects. It is therefore of the utmost importance that the information they share with their stakeholders, through their website, communicates their plans for growth and the potential return on investment. 
Porter (2001:64) recognises the importance of considering corporate strategies when designing the website strategy. He identifies principles that organisations could use as guidelines when positioning the website strategy in the organisation to ensure that it complements the corporate strategies. Gakhar (2012:74) states that an effective alignment of the website strategy to the organisational strategies increases investment opportunities for the organisation and potential customers. The alignment plays a vital role in the decision-making process of the potential investor and determines the outcome of the decision-making process.

\section{Investment criteria used by investors and funders}

According to Kollman and Kuckertz (2010:5) there are investors who raise money and then look for an organisation that has the potential to yield a high return on their investment. The investors engage in investment processes where the deal origination is determined. The deal is screened, evaluated and the structure of the investment is analysed to identify whether or not the structure will support the execution of the project at hand (Kollman \& Kuckertz 2010:9). Once the investment decision has been made, the investor will monitor the performance of the organisation to ensure that the goals and objectives that were set are being executed effectively. The organisation is constantly monitored to determine whether it is producing the desired results or not. There is an additional set of investment criteria which will be discussed in the ensuing section.

When investing in organisations, an investment criterion is used to evaluate the potential of the organisations that seek investment opportunities. Kollman and Kuchertz (2010:27) have identified the factors that investors consider before investing in an organisation or providing funds that will facilitate for completion of the organisation's projects. Below is a discussion of these factors:

- Personalities of the entrepreneurs: the skills, capabilities and competencies of the leaders of the organisation are assessed based on the achievements of the organisation under their leadership. Leaders play influential roles in the behaviours and attitudes of employees by setting the culture that filters throughout the organisation. The business practices that are employed by the leaders are assessed to see whether or not they are ethical and whether they are efficient in achieving the goals and objectives or not. The reputation of leaders is also assessed to reduce risks of losing returns as a result of the leader reputation-factor.

- Experiences of entrepreneurs: investors assess the organisation's growth and the development that has taken place over a certain period of time by looking at its track record. There are activities and business practices that require organisations to be accredited or qualified for practice. The investor evaluates the business qualifications and the technical qualifications of the organisation and its staff to ensure they have the relevant experience.
- Products or services: the needs of customers continuously evolve and require organisations to be flexible, innovative and responsive to those needs. Investors assess products and services and identify how the organisation adds value to the final product. The organisation claims its competitive advantage from its unique selling point, core competencies and capabilities which are assessed to identify whether or not they will be able to sustain the innovation rate.

- Market characteristics: the potential risks and threats that reside in the market of the target investment are identified and assigned weights to calculate the potential impact of a threat. The potential growth of the market is also assessed by measuring how the market accepts new products and services. The market players and external factors that influence the operation of the organisation are also identified and assessed.

- Financial characteristics: the investor assesses the potential return on investment when considering an investment in the specific organisation. The financial characteristics of the organisation determine whether or not the investor will invest in the organisation or will seek other investment opportunities (Kollman \& Kuchertz 2010:27).

The above-mentioned investment criteria are factors that were considered when the strategic objective measurement indicators were developed to assess Aurum's website.

There are different types of investors, which include, firstly, international investors who are located in a different country from where they invest. Secondly, the other type of investor is the domestic investor who resides within the same country in which the investment lies (Kollman \& Kuchertz 2010:15). International and local investors who seek investment opportunities often make use of Internet technologies to access different websites of organisations that seek investment opportunities. The content quality, the accessibility, navigability and the downloading speed of the website will determine whether the investor is more likely or not to invest in the organisation. Organisations make use of websites as a medium that can transfer information and knowledge which can facilitate positive outcomes for the decision-making process followed by the investors (BouLlusar \& Segarra-Cipres 2006:102).

\section{Methodology}

The Aurum Institute was used as a case study to examine the factors that determine the quality of a website's content and how information and knowledge is shared by the organisation with its customers and stakeholders. Secondary data was analysed which included the outcome of Aurum's knowledge audit (which was performed one year prior to this study by Aurum's knowledge manager), Aurum's website content as well as their organisational strategy document. Liebowitz et al. (2000:3) defines a knowledge audit as a tool appropriate to evaluate how the organisation would need to be changed to better facilitate for the flow and sharing of information and knowledge. The website content 
and the strategy document were compared to identify any discrepancies that existed between the two data components.

Data was collected using an observation schedule to assess the characteristics of Aurum's website and to evaluate the content. As a result of the dynamic nature of the website, where regular changes are made, the content was captured, for a period of one week, for website observation. This approach is in line with the principles of 'one-way-mirror' research to allow the analysis of website content and secondary data without any alterations of the content (Healy \& Perry 2000:119). This approach allowed the authors to provide a description of what was experienced and measure that which was observed (Krauss 2005:760).

A qualitative approach to collecting and analysing data was employed to produce the findings for the research problems identified. The qualitative method of collecting data provided the authors with descriptive data, which facilitated processing and the unearthing of findings that are in-depth and descriptive. This type of research is empirical, according to Wacker (2008:10), because the study's expected results or findings are used as inputs into the designing processes of the prototype website, which will represent the desired website strategy. Secondary data were collected for analysis, which included Aurum's organisational strategy document as well as their website content. The website was analysed and compared to the organisational strategies to identify the gaps. After the analysis of the secondary data, an interview with the knowledge manager was conducted. This was to allow for the validation, triangulation and clarification of the data findings and insight regarding the assessment of the website content against the organisational strategy. The interview also clarified matters for the development of a prototype website. The nature of the data prescribed the descriptive analysis techniques for the analysis of secondary data.

Coding was used to analyse the secondary data qualitatively. This analysis was applied to identify the associations between the different aspects of the data or codes using a relationship analysis technique, as identified by Saldaña (2009:3). The collection and analysis of data were performed with the aim of creating a website prototype that will successfully compliment the organisational strategic objectives of Aurum, and potentially increase investment opportunities. The organisational strategic objectives were translated into measurement indicators, and used as benchmarks to measure how each webpage on the website represented each of the identified objectives.

Ethical practices were considered in the collection and analysis of data. The Aurum Institute's strategic objectives that were used in the secondary data analysis, are confidential and have strategic value to the organisation. Disclosing this information may jeopardise the success of the organisation and its projects and therefore the data analysis used undisclosed codes. In the interview session the information of the participant remained confidential, and permission was requested from the organisation to conduct the research. The highest technical skills and methodologies were employed in handling the data to avoid fabrication of the data.

Limitations that were encountered included the fact that Aurum's website content was subject to change at any given moment. The results of the content analysis only apply to a specific period of time as the website content has since been updated, edited or removed. However, the lessons learnt from the findings remain applicable from a holistic perspective regardless of editorial and maintenance updates.

\section{Findings: Website content representative of organisational goals}

Descriptive analytical tools were used to identify the patterns and the relationships between the organisational website and the corporate strategic objectives from the identified sources of secondary data.

The results, from the analysis, were used as inputs in the designing and development of the website prototype. The

TABLE 1: Representation of organisational strategic objectives by each webpage.

\begin{tabular}{|c|c|c|c|c|c|c|c|}
\hline \multirow[t]{2}{*}{ Webpage visited } & \multicolumn{6}{|c|}{ Objective } & \multirow[t]{2}{*}{ Total objectives per page } \\
\hline & 1 & 2 & 3 & 4 & 5 & 6 & \\
\hline Home page & 3 & 2 & 1 & 3 & 3 & 3 & 15 \\
\hline Management team & 1 & 1 & 1 & 3 & 1 & 3 & 10 \\
\hline Aurum in the news & 3 & 1 & 1 & 3 & 1 & 1 & 10 \\
\hline Aurum events & 3 & 3 & 1 & 3 & 3 & 3 & 16 \\
\hline Aurum staff awards & 1 & 1 & 1 & 1 & 1 & 1 & 6 \\
\hline Career opportunities & 1 & 1 & 1 & 1 & 1 & 3 & 8 \\
\hline Aurum training & 3 & 2 & 1 & 3 & 1 & 3 & 13 \\
\hline Scholarships & 1 & 1 & 1 & 3 & 1 & 3 & 10 \\
\hline Publications & 1 & 3 & 1 & 1 & 1 & 3 & 10 \\
\hline Funders & 1 & 1 & 1 & 3 & 1 & 1 & 8 \\
\hline Collaborators & 1 & 1 & 1 & 3 & 3 & 1 & 10 \\
\hline Contact us & 1 & 1 & 1 & 2 & 1 & 1 & 7 \\
\hline Total representativeness of website & - & - & - & - & - & - & $123 / 213$ \\
\hline
\end{tabular}

Keys: 1 = Hidden; 2 = Obscure; 3 = Visible 
first prototype presentation was based on a gap analysis that resided between the website and the organisational strategic objectives.

The strategic objectives were translated into measuring indicators against the website to identify how each webpage represents the strategic objectives. Table 1 illustrates how each objective was represented by each of the webpages.

After the development of the first prototype, an interview was conducted with Aurum's knowledge manager and aligned to the knowledge audit that was conducted prior to this study. This interview also triangulated the requirements necessary to successfully represent the organisational strategic objectives. The feedback received from the interview was incorporated into the re-designing stage of the prototype which was then used as recommendations to the organisation.

Table 1 illustrates the analysis of Aurum's website content against the organisational strategic objectives that were used as the measuring indicators. The webpages were individually assigned weights using a three-point scale which determined whether or not the strategic objectives were (1) not effectively represented or hidden, (2) obscure or ambiguous, or (3) visible and clear. The highest total that a webpage could score was 18. The maximum possible weight of 213 was calculated by multiplying the six objectives with the highest possible weight ( 3 - visible) that the webpage could score per objective. Each webpage was given a total weight out of a possible maximum of 18 (webpage weight is the sum of all the points attained from each objective per webpage). The results derived from the analysis of the findings presented in Table 1 are subsequently discussed:

- Home page: This page was allocated a score of 15 out of 18 , meaning it is $83 \%$ representative of Aurum's strategic objectives. This webpage achieved this weighting score by including information regarding disease management with specific focus on prevention, treatment and care of the communities. The programs that the institute engaged in were also covered and included on the home page. The home page is the information users' first point of contact with the organisation's content, therefore a score of $83 \%$ indicates that it is more representative of Aurum's strategic objectives. This high representativeness enables the information user to obtain a clear understanding of the organisational strategic objectives and gives funders an idea of projects they could potentially sponsor.

- Management team: This page was allocated a score of 10 out of 18 , meaning it is $56 \%$ representative of Aurum's strategic objectives. This was achieved by including information about the staff responsible for leading Aurum. Information on their professional qualifications was also made available. This information enables the potential funder to gain insight in the credibility of the management team that leads the organisation.
- Aurum in the news: This page was allocated a score of 10 out of 18 , meaning it is $56 \%$ representative of Aurum's strategic objectives. This was achieved by the page's inclusion of the various news articles and information on community work carried out around the country. This information is vital as it could assist potential investors to assess the publicity and reputation of Aurum.

- Aurum events: This page was allocated a score of 16 out of 18 , which indicates that it is $89 \%$ representative of Aurum's strategic objectives. This representation was achieved by the page's inclusion of information about the events that Aurum has hosted and projects it undertook. This information is relevant for potential investors as they are able to see the outcomes of investments made by other funders. This thus gives them an idea of how their funding would be spent should they decide to invest in Aurum.

- Aurum staff awards: This page was allocated a score of 6 out of 18; this shows that it is 33\% representative of Aurum's strategic objectives. This was achieved through the page's inclusion of information on the awards that were given to Aurum's staff who performed exceptionally well. Although this information adds value, and could be useful for potential investors by enabling them to identify the skills and abilities of the Aurum staff, this page should be on the institute's intranet and not the website. This is because it is more focused on internal staff affairs and internal recognition of performance.

- Career opportunities: This page was allocated a score of 8 out of 18, meaning it is $44 \%$ representative of Aurum's strategic objectives. This was evident in the page's inclusion of information on the vacancies that are currently available at the Aurum Institute, together with a description of the vacant positions. The $44 \%$ is understandably low, resulting from the nature of the page.

- Aurum training: This page was allocated a score of 13 out of 18 , meaning it is $72 \%$ representative of Aurum's strategic objectives. This was accomplished by the page's inclusion of information on the clinical training, counselling training courses, seminars, bookings for training, downloads and past programs. This information is very important for potential investors as it provides insight into the quality of the curriculum offered by the Aurum Institute.

- Scholarships: This page was allocated a score of 10 out of 18 , meaning it is $56 \%$ representative of Aurum's strategic objectives. This was achieved by including information on the scholarships provided by the organisation, which demonstrates a corporate social responsibility on Aurum's part, through provision of education or skills development programs. This attempt by Aurum to empower the community (corporate social responsibility) is likely to attract potential investors.

- Publications: This page was allocated a score of 10 out of 18 , meaning it is $56 \%$ representative of Aurum's strategic objectives. It included information on topics of interest 
including the prevention, treatment and control of the HIV and TB pandemic. This page's representativeness percentage should be higher given that Aurum is a health research institute. Together with the research available, information should be provided on how this research will help Aurum in eradicating the scourge of TB and HIV and AIDS.

- Funders: This page was allocated a score of 8 out of 18, meaning it is $44 \%$ representative of Aurum's strategic objectives. It contained information on the funders who have worked with the organisation. This representativeness percentage should be higher as this information should give potential investors the confidence and security to dedicate resources to the Aurum Institute.

- Collaborators: This page was allocated a score of 10 out of 18, meaning it is 56\% representative of Aurum's strategic objectives. This was achieved through the inclusion of information on those organisations, both international and domestic, that the Aurum institute works with to successfully improve the welfare of communities. This page's representativeness should also be higher, given that it is one of the pages that are most likely to be visited by potential investors.

- Contact us: This page was allocated a score of 7 out of 18 , meaning it is 39\% representative of Aurum's strategic objectives. It included Aurum's contact details at head office as well as the addresses of the different branches. The nature of this page allows it to score in this percentage as it is generally not a content heavy page.

Based on the results presented above, it is now known that Aurum's website content, as a whole, is $57.7 \%$ representative of the institute's strategic objectives. The percentage was calculated by dividing the sum total of all the webpages (123) with the maximum total that the webpage could score (213). From the measurement indicators used on the individual webpages, it was illustrated how the website achieved this percentage. A result of $57.7 \%$ indicates that Aurum's website content does not successfully communicate the organisational strategic objectives. The analysis of the results above also offers a clear indication of the webpages on which the organisation's objectives should have or have been displayed.

\section{Conclusion}

The significance of this study was to establish the extent to which Aurum's website content is aligned to the organisation's strategic objectives. Based on the research findings it is evident that Aurum's website content is not effectively representative of these strategic objectives. The reason for this could be attributed to information overload, insufficiency in relevant information and the presentation of content. These issues could be negatively affecting the funding, causing the difficulties that Aurum currently faces. It has been noted, however, that some of the webpages are not meant to contain too much information and, thus, contributed to the low percentage that Aurum scored in its overall representativeness of the strategic objectives.

The theoretical approach that was taken can be utilised to assess the websites of other organisations that aim to achieve similar results. The results from this study are appropriate for application to other NPOs, as well as for-profit organisations, to analyse their website content. During the literature review phase of this study a shortage of academic research, on the importance of website content alignment to the strategic objectives of an organisation, was realised. Further research must be conducted on this topic, particularly for non-profit organisations, as this has an impact on the potential funding that they could attract. Additional research will need to be conducted on the measurement of the funding that the Aurum Institute would attract, once their website content has been aligned to the strategic objectives of the organisation. In other words, two types of website should be measured and compared for how effective each is at attracting funding or sponsorships, one which has website content that is aligned to its organisational strategic objectives, in comparison to one that is not thus aligned. This would require a longitudinal research approach.

The processes of website planning and defining the purpose of the website content should be largely influenced by the organisational strategic goals of the Aurum Institute, to ensure that relevant content is placed on the website. Aligning Aurum's website content with the strategic objectives of Aurum will enable the organisation to effectively communicate information that is relevant to the target audience (potential investors), and will successfully facilitate quick and positive decision-making by the potential investor. The factors that constitute a successful website strategy include the loading speed, navigability, ease of access, and available content. These factors are most likely to influence the decision-making processes of stakeholders and potential investors. Further research opportunities exist to identify a model that will evaluate the information needs of investors.

This study provides Aurum with insight, on the specific aspects of their website that are currently lacking, for improved communication of its organisational objectives. The analysis shows the webpages where the organisation's objectives are visible, and where they are not, but should be visible. Furthermore, the website can now be developed into an effective platform that effectively reflects the essence of the organisation, in order to specifically gain the attention of potential funders who would need to know whether or not their funding intent is directed at the correct funding recipient.

It can be concluded that when a website's content is aligned to the strategic objectives of the organisation, and does so on a website structure that is easy to navigate and presents the information in an easily consumable manner, the website could be effective in drawing the attention of the user, which could ultimately translate into increased and improved funding. 


\section{Acknowledgements Competing interests}

The authors declare that they have no financial or personal relationship(s) that may have inappropriately influenced them in writing this article.

\section{Authors' contributions}

K.M. (University of Johannesburg) and M.R. (University of Johannesburg) conducted the research as postgraduate students under the supervision of M.M. (University of Johannesburg). M.M. conceptualised the project and K.M. and M.R. collected and analysed the relevant data with supervision support from M.M.

\section{References}

Akakandelwa, A., 2011, 'An exploratory survey of the SADC e-government website', Library Review 60(5), 421-431. http://dx.doi.org/10.1108/00242531111135317

Aurum Institute, 2010, 'Annual Report 2010', viewed 18 August 2012, from http:// www.auruminstitute.org/phocadownload/annual_report_2010.pdf

Aurum Institute, 2012, 'Company profile', viewed 04 August 2012, from http://www. auruminstitute.org/publications.php

Bailey, C., 2010, 'Content is king by Bill Gates', viewed 12 September 2012, from http://www.craigbailey.net/content-is-king-by-bill-gates.html

Baloglu, S. \& Pekcan, Y.A., 2006, 'The website design and internet site marketing practices of upscale and luxury hotels in Turkey', Tourism Management 27, 171176. http://dx.doi.org/10.1016/j.tourman.2004.07.003

Bou-Llusar, J.C. \& Segarra-Cipres, M., 2006, 'Strategic knowledge transfer and its implications for competitive advantage: an integrative conceptual framework', Journal of Knowledge Management 10(4), 100-112. http://dx.doi. org/10.1108/13673270610679390

Chiou, C.W., Lin, C. \& Perng, C., 2010, 'A strategic framework for website evaluation based on the review of the literature from 1995-2006', Information and Management 47, 282-290. http://dx.doi.org/10.1016/j.im.2010.06.002

Chiou, C.W., Lin, C. \& Perng, C., 2011, 'A strategic website evaluation of online trave agencies', Tourism Management 32(6), 1463-1473. http://dx.doi.org/10.1016/j. tourman.2010.12.007

De Troyer, O., 1998, Designing well-structured websites: Lessons to be learned from database schema methodology, Tilburg University, Tilburg.

Dias, I. \& Reinhard, N., 2004, 'Methodology for web presence strategy formulation: A case study', Proceedings 17th Bled e-Commerce Conference, Slovenia, 21-23 June, Brazil.

Gakhar, D.V., 2012, 'Perception of stakeholders on web-based corporate reporting practices', Journal of Advances in Management Research 9(1), 64-76.
Gates, B., 1996, 'Content is king', Wordpress, San Francisco.

Grigoroudis, E., Litos, C., Moustakis, V., Politis, Y. \& Tsironis, L., 2008, 'The assessment of user-perceived web quality: application of a satisfaction benchmarking approach', European Journal of Operational Research 187(3), 1346-1357. http:// dx.doi.org/10.1016/j.ejor.2006.09.017

Healy, M. \& Perry, C., 2000, 'Comprehensive criteria to judge validity and reliability of qualitative research within the realism paradigm', Qualitative Market Research - An International Journal 3(3), 118-126. http://dx.doi. org/10.1108/13522750010333861

Hernandez, B., Jimenez, J. \& Martin, M.J., 2009, 'Key website factors in e-business strategy' International Journal of Information Management 29, 362-371. http:// dx.doi.org/10.1016/j.ijinfomgt.2008.12.006

Huizingh, E., 2000, 'The content and design of websites: an empirical study', Information \& Management 37, 123-134. http://dx.doi.org/10.1016/S03787206(99)00044-0

Hussainey, K. \& Mouselli, S., 2010, 'Disclosure quality and stock returns in the UK', Journal of Applied Accounting Research 11(2), 154-174. http://dx.doi. org/10.1108/09675421011069513

Kollman, T. \& Kuckertz, A., 2010, 'Evaluation uncertainty of venture capitalists' investment criteria', Journal of Business Research 63, 741-747. http://dx.doi. org/10.1016/j.jbusres.2009.06.004

Krauss, S.E., 2005, 'Research paradigms and meaning: A primer', The Qualitative Report 10(4), 758-770.

Liao, H., Proctor, R. W. \& Salvendy, G., 2008, 'Content preparation for cross-cultural e-commerce: a review and a model', Behaviour and Information Technology 27(1), 43-61. http://dx.doi.org/10.1080/01449290601088424

Liebowitz, J., Rubenstein-Montano, B., McCaw, D., Buchwalter, J. \& Browning, C., 2000, 'The knowledge audit', Knowledge and process management $7(1)$ 3-10. http://dx.doi.org/10.1002/(SICI)1099-1441(200001/03)7:1\%3C3::AIDKPM72\%3E3.0.CO;2-0

Nacar, R. \& Burnaz, S., 2011, 'A cultural content analysis of multinational companies' websites', Qualitative market research: An international Journal 14(3), 274-288. $\mathrm{http}: / /$ dx.doi.org/10.1108/13522751111137505

Moustakis, V., Tsironis, L. \& Litos, C., 2006, 'A model of website quality assessment', The Quality Management Journal 13(2), 22-37.

Mpindiwa, L., 2010, 'Impact of intranet as a knowledge sharing tool: experiences of ZIMRA', World Library and Infromation Congress: 76th IFLA General Conference and Assembly, Gothernburg, Sweden.

Panopoulou, E., Tambouris, E. \& Tarabanis, K., 2008, 'A framework for evaluating websites of public authorities', Aslib Proceedings 60(5), 517-546. http://dx.doi. org/10.1108/00012530810908229

Porter, M., 2001, 'Strategy and the internet', Harvard Business Review, March, 1-20.

Rocha, A., 2012, 'Framework for a global quality evaluation of a website', Online Information Review 36(3), 374-382. http://dx.doi. org/10.1108/14684521211241404

Saldaña, J., 2009, The coding manual for qualitative researchers, Sage, London.

Schmidt, S., Cantallops, A.S. \& Dos Santos, C.P., 2008, 'The characteristics of hotel websites and their implications for website effectiveness', International Journal of Hospitality Management 27, 2008, 504-516. http://dx.doi.org/10.1016/j. ijhm.2007.08.002

Wacker, J.G., 2008, 'Tragic conceptual flaws of theory - building empirical research', Decision Line, October, 10-13. 